\title{
The role of oxygen atoms on the excitons at the
}

\section{edges of monolayer $\mathrm{WS}_{2}$}

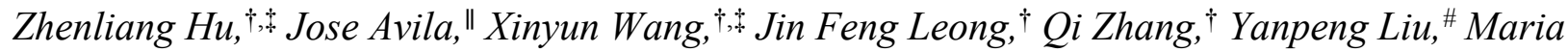

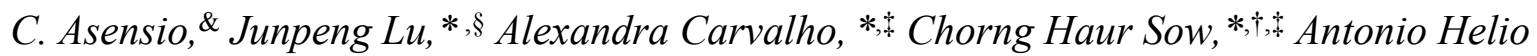
Castro Neto $*, \dagger,+$

†Department of Physics, National University of Singapore, 2 Science Drive 3, 117542 Singapore Center for Advanced 2D Materials, National University of Singapore, 6 Science Drive 2,

117546 Singapore

"ANTARES Beamline, Synchrotron SOLEIL \& Université Paris-Saclay, L’Orme des Merisiers, Gif sur Yvette CEDEX, France

\#Key Laboratory for Intelligent Nano Materials and Devices of Ministry of Education, and Institute of Nanoscience, Nanjing University of Aeronautics and Astronautics, Nanjing 210016, China

\&Instituto de Materiales de Madrid, CSIC, C/ Sor Juana Inés de la Cruz, 3 Cantoblanco, 28049

Madrid, Spain

${ }^{\S}$ School of Physics, Southeast University, 2 Southeast University Road, Nanjing 211189, China

\section{Corresponding Authors}

*E-mail: phyljp@seu.edu.cn; Fax: 025-52090600-8201 (J.L.)

*E-mail: carvalho@sapo.tl; Fax: (65) 6516 1161(A.C.)

*E-mail: physowch@nus.edu.sg; Fax: (65) 65162957 (C.H.S.)

*E-mail: phycastr@nus.edu.sg; Fax: (65) 66012575 (A.H.C.N.) 

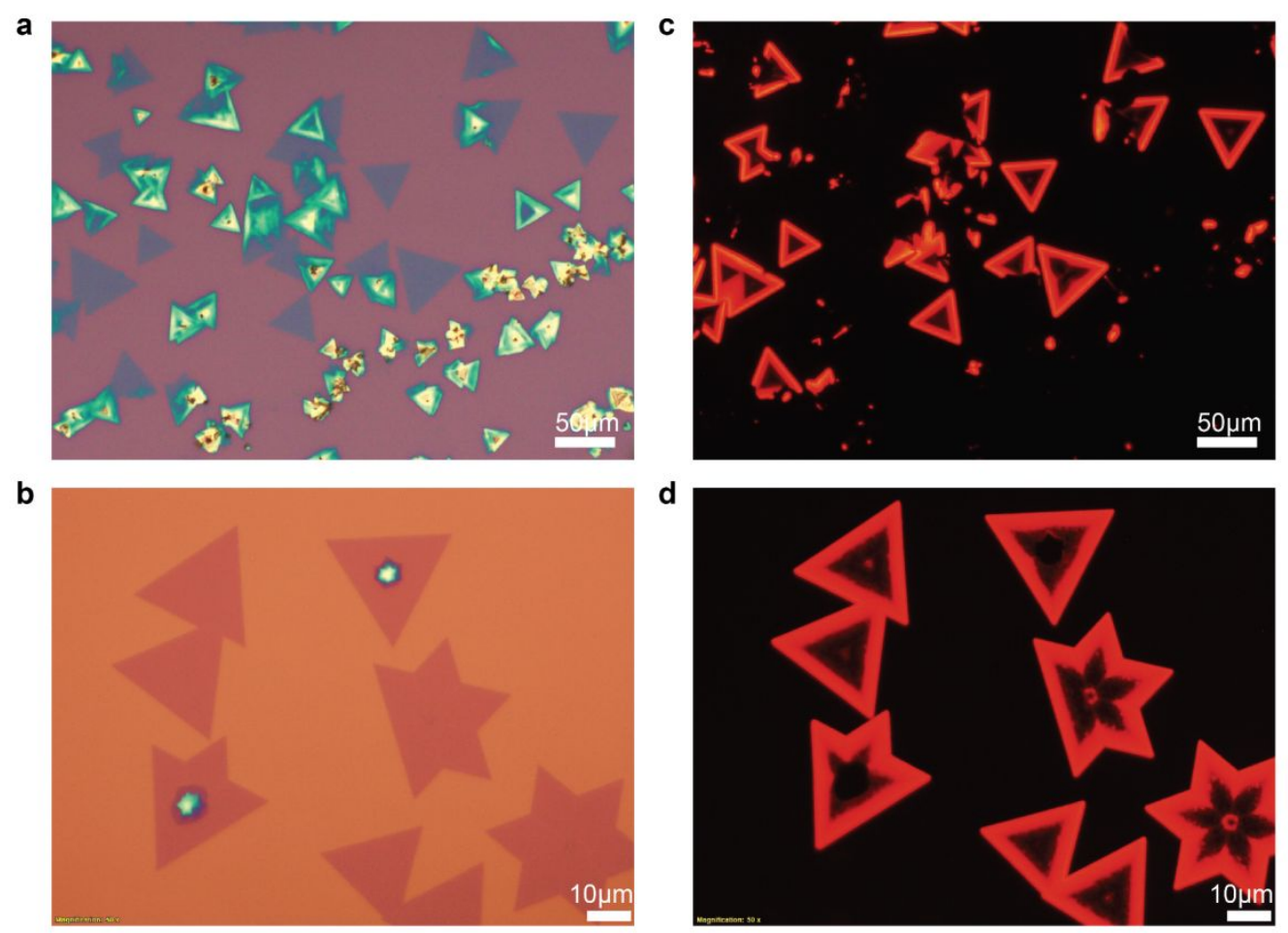

Figure S1. $\mathrm{WS}_{2}$ monolayers with edge fluorescence enhancement. (a, b) Optical images of $\mathrm{WS}_{2}$ monolayers which are grown on $\mathrm{Si} / \mathrm{SiO}_{2}$ substrate. (c, d) Fluorescence images of the respective $\mathrm{WS}_{2}$ monolayers. All the monolayers display the edge fluorescence. Note that the fluorescence images were taken under the yellow excitations with excitation time around five seconds. 


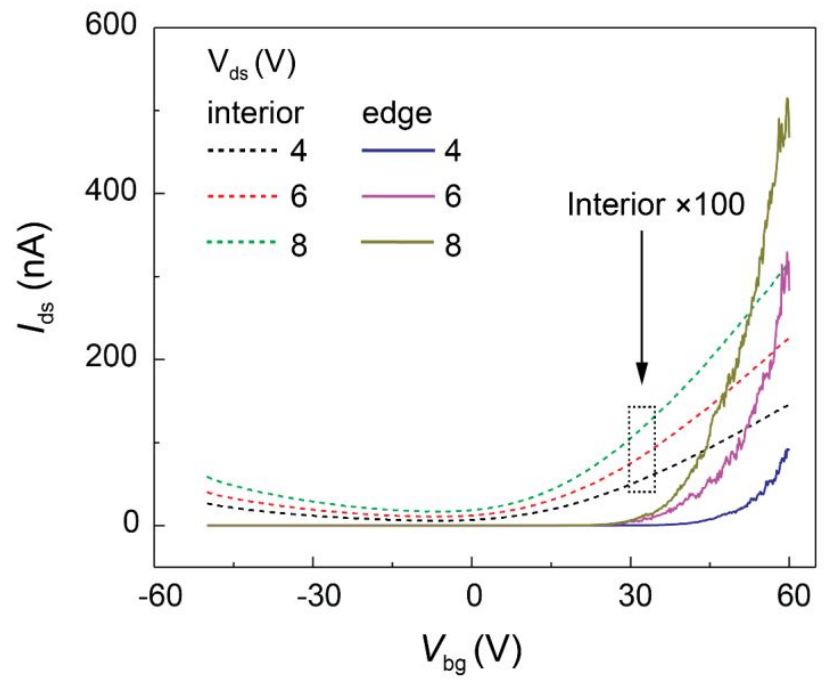

Figure S2. Two-probe transport curves of the WS2 edge-ribbon based and interior-ribbon based transistors. The source-drain bias is fixed at $4 \mathrm{~V}, 6 \mathrm{~V}$ and $8 \mathrm{~V}$, separately. To have a better comparison, the currents of the interior-ribbon based transistor have been multiplied by 100 .
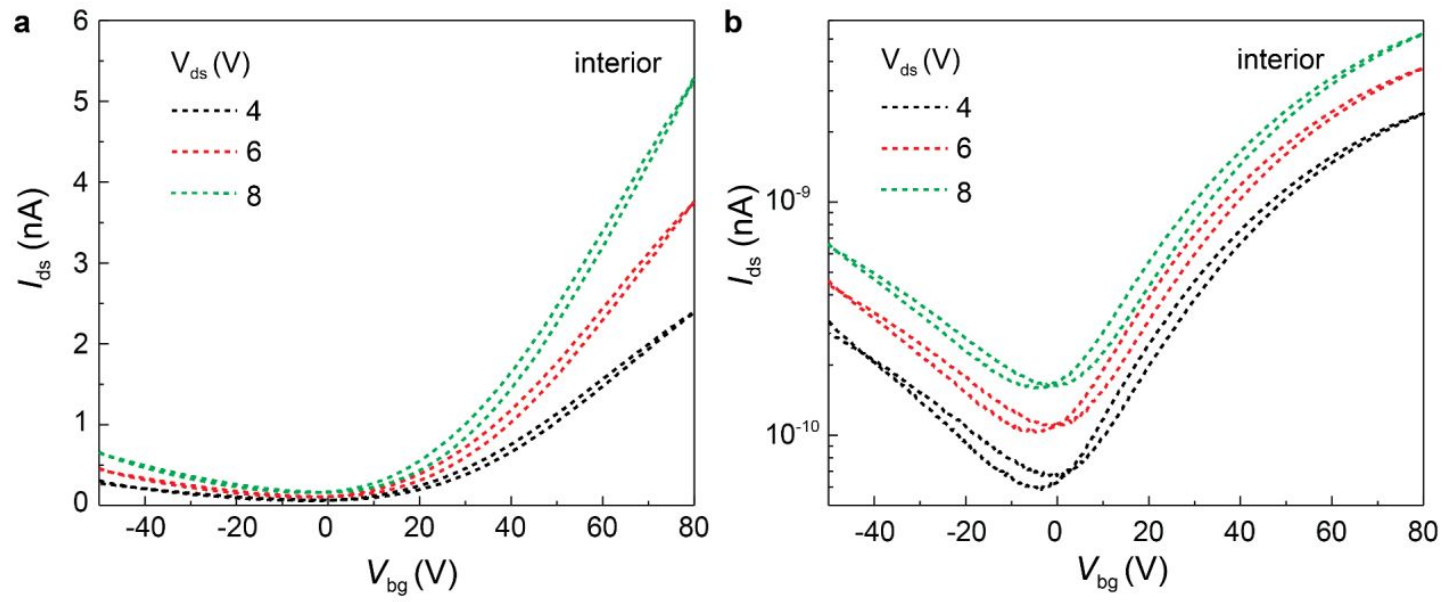

Figure S3. The transport curves of interior-ribbon based transistor in linear scale (a) and semilogarithmic scale (b). Even though the gate voltage was increased to $80 \mathrm{~V}$, the transistor was still at the off state. It indicates that the threshold voltage of the interior-ribbon based transistor is higher than $80 \mathrm{~V}$. 


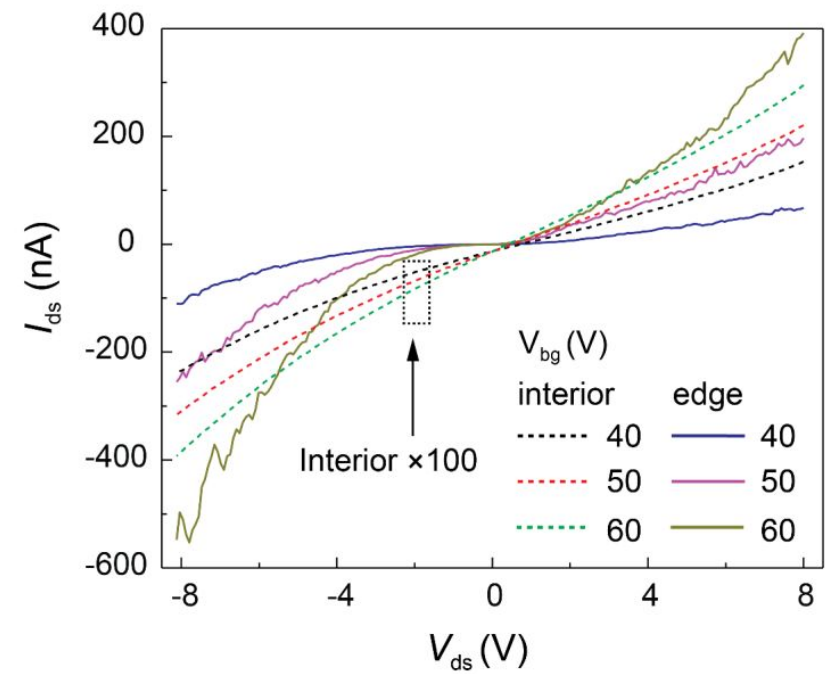

Figure S4. The electrical conduction curves of two different transistors. For the edge-ribbon based transistor, the source-drain current is strongly nonlinear to the source-drain bias. While for the interiorribbon based transistor, the current is almost linear to the bias.
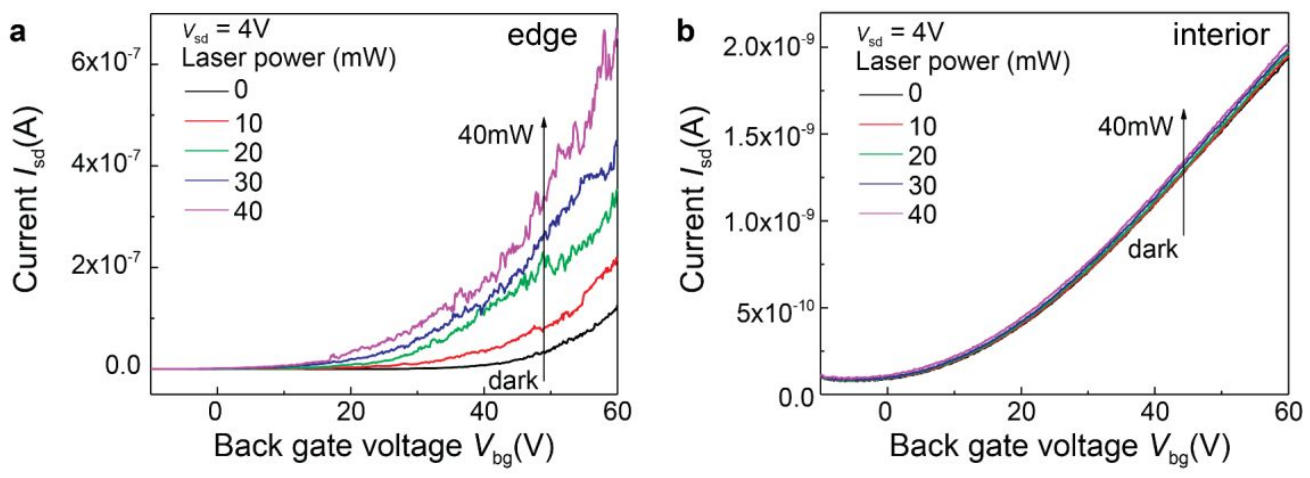

Figure S5. Transport curve of two transistors when illuminated under 532nm laser with different power. The laser strongly increased the performance of the edge-ribbon based transistor (a), while it had no effect on the interior-ribbon based transistor (b). The size of the laser spot is around $7 \mathrm{~mm}^{2}$. 

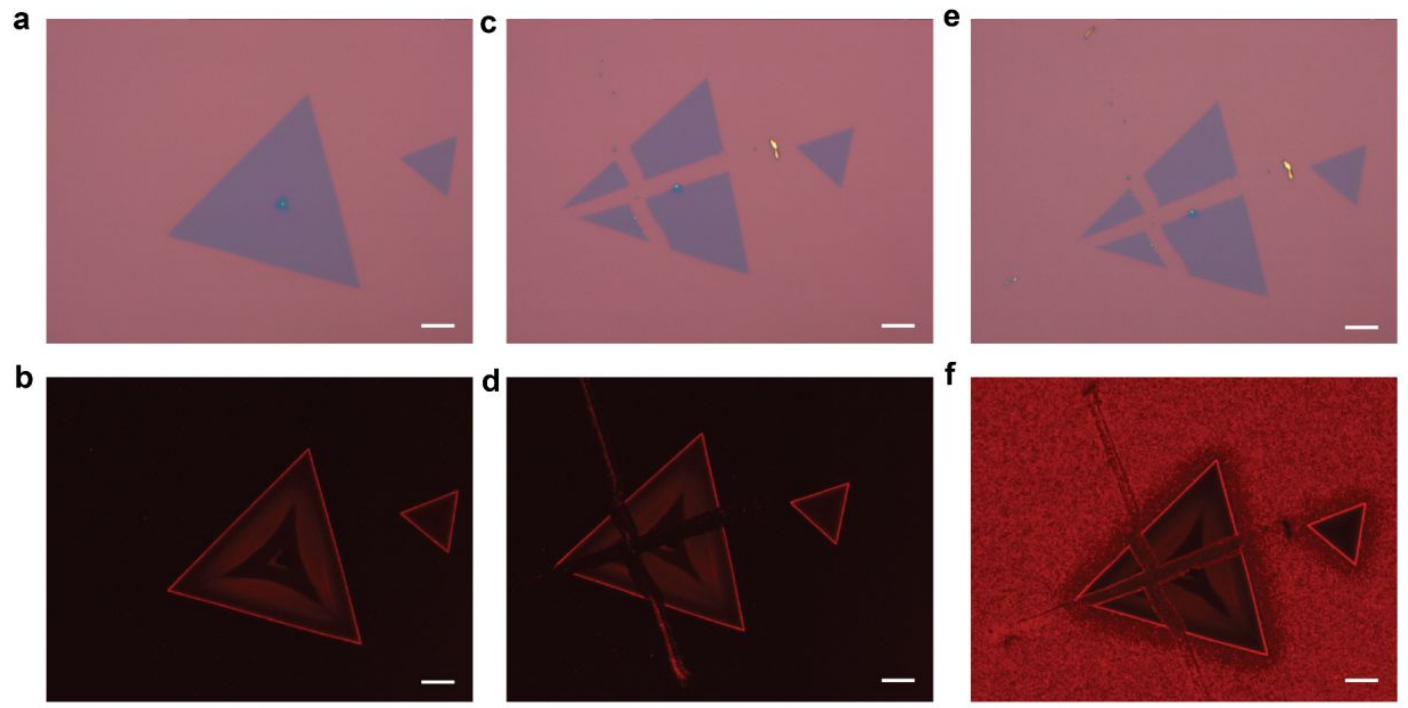

Figure S6. Effect of the oxygen annealing on the fresh edges of $\mathrm{WS}_{2}$ monolayer. (a, b) Optical and fluorescence images of the as-grown $\mathrm{WS}_{2}$ monolayer. sharp and bright edges are displayed. (c, d) Optical and fluorescence images of $\mathrm{WS}_{2}$ monolayer with the mechanically scratched edges. (e, f) Optical and fluorescence images of the $\mathrm{WS}_{2}$ monolayer with scratched edges after thermal annealing in $\mathrm{O}_{2}$ environment. Scratched edges exhibited significant fluorescence after thermal annealing in $\mathrm{O}_{2}$ environment. Scale bar is $10 \mu \mathrm{m}$.
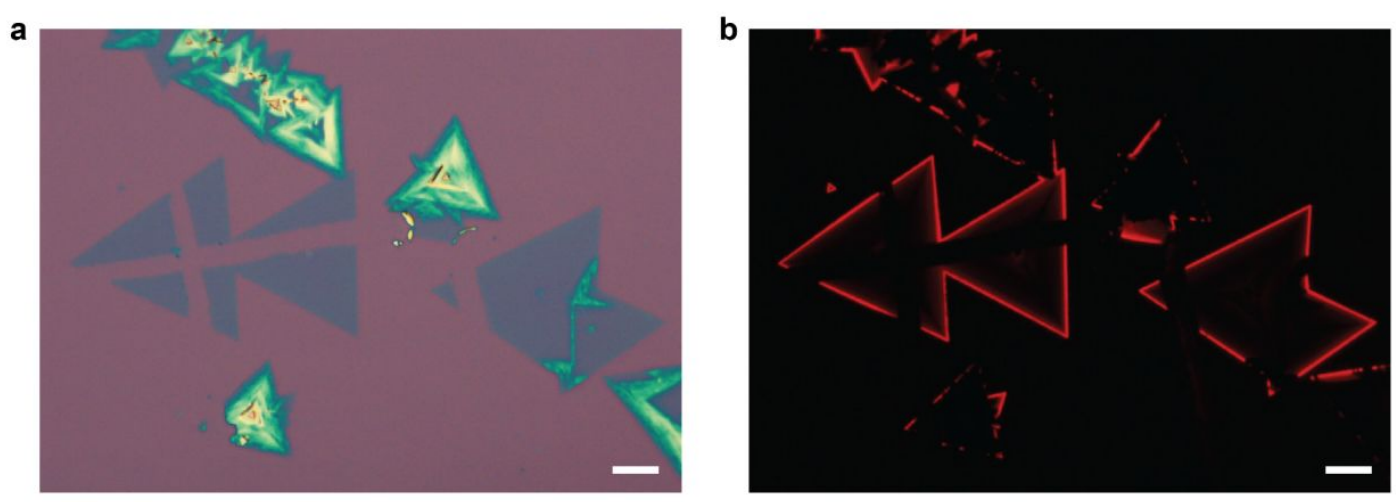

Figure S7. Optical (a) and fluorescence (b) image of $\mathrm{WS}_{2}$ monolayer with scratched edges after thermal annealing in inert environment. No significant edge fluorescence at the scratched edges were displayed. Scale bar is $10 \mu \mathrm{m}$. 
a

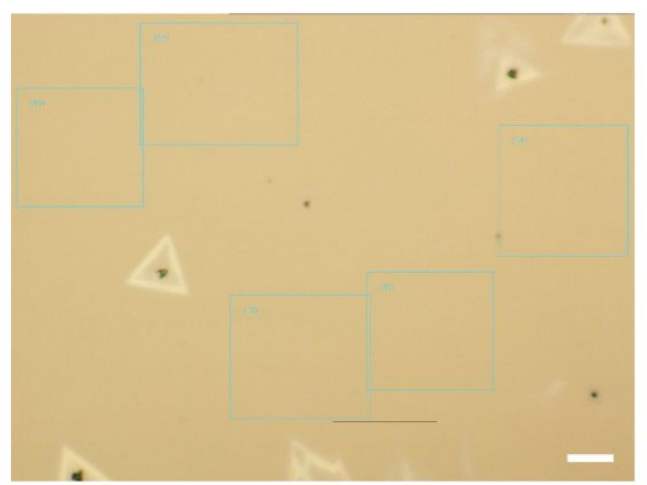

b

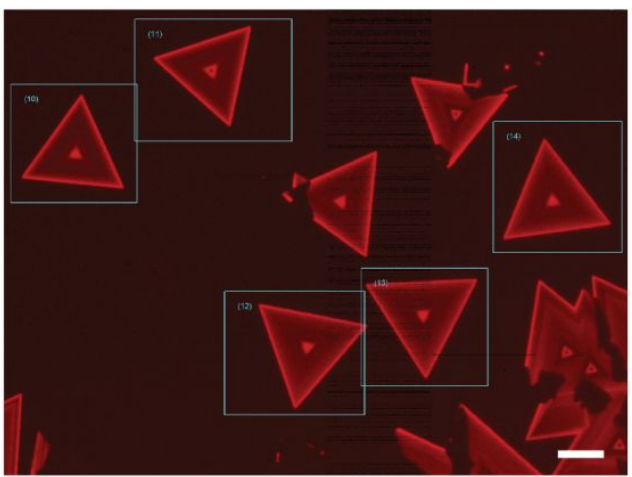

Figure S8. Optical (a) and fluorescence (b) image of $\mathrm{WS}_{2}$ monolayer grown on highly n-doped Si substrates. Because of the contrast, it is very hard to observe the $\mathrm{WS}_{2}$ monolayer on Si substrate under optical microscope. Rectangles mark the position of $\mathrm{WS}_{2}$ monolayers. Scale bar is $10 \mu \mathrm{m}$.

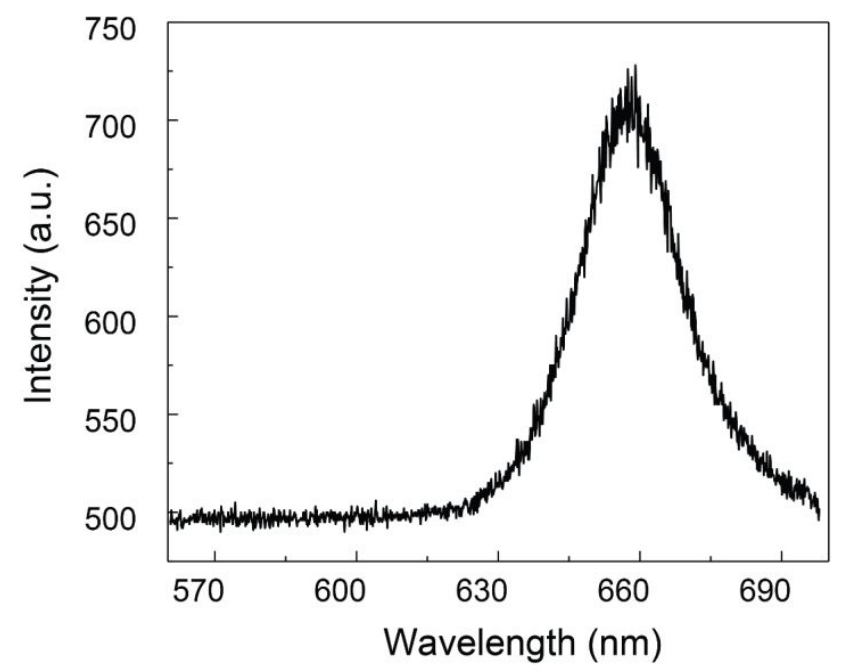

Figure S9. PL spectrum in central triangle. The peak is around $660 \mathrm{~nm}(1.88 \mathrm{eV})$, and full width at half maximum is around $80 \mathrm{meV}$. 


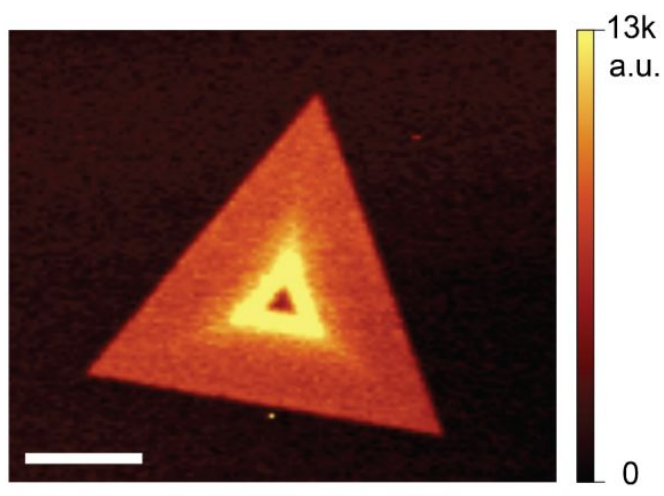

Figure S10. Raman mapping of the $\mathrm{A}_{1 \mathrm{~g}}$ peak of WS2 monolayer. The integration range is from $400-430$ $\mathrm{cm}^{-1}$. Scale bar is $7 \mu \mathrm{m}$.
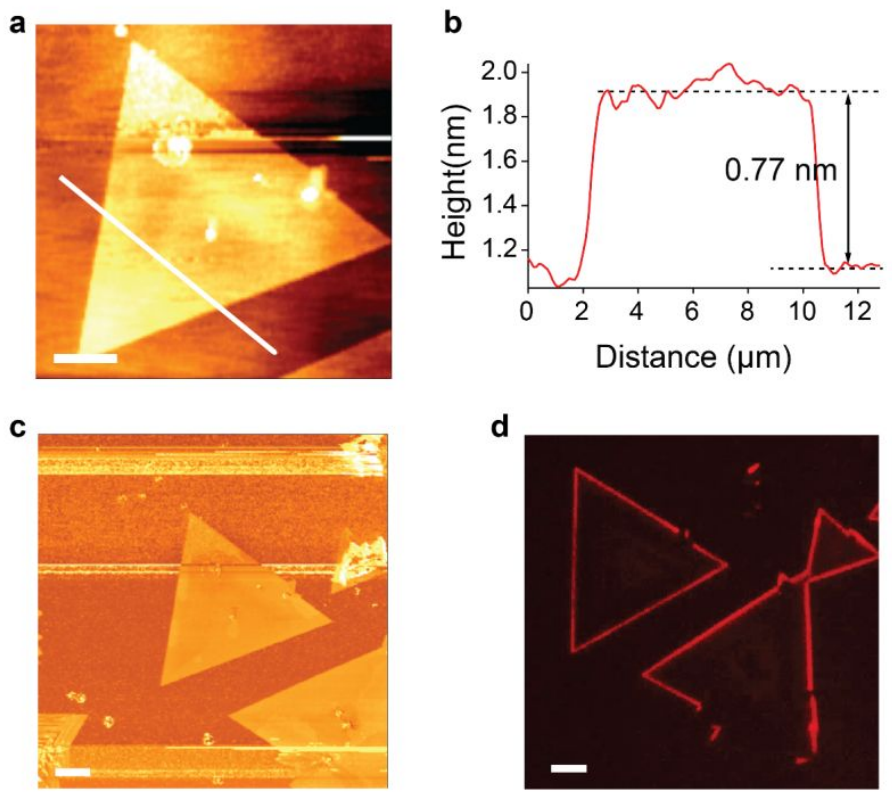

Figure S11. AFM height and phase image of $\mathrm{WS}_{2}$ monolayer on Si substrate. (a, b) AFM height image and height profile along the white line show that the measured $\mathrm{WS}_{2}$ flake is single layer. (c) AFM phase image shows no obvious distinction over the whole flake. (d) The fluorescence image furtherly helps us to confirm the single layer $\mathrm{WS}_{2}$. Scale bar is $5 \mu \mathrm{m}$. 


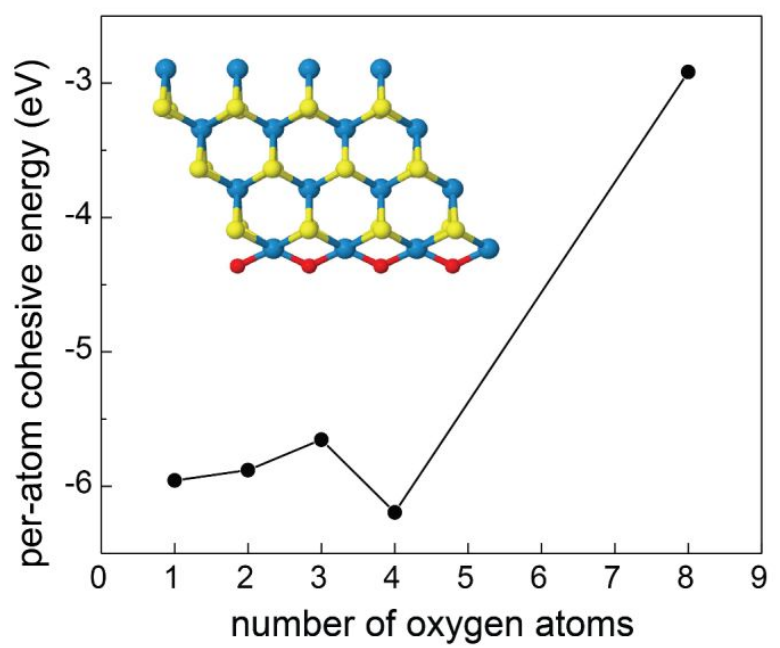

Figure S12. Stability study of the simulated structures. The stability of the structure is evaluated by calculating the cohesive energy of the same chemical configuration but with different number of oxygen atoms chemisorbed at the edge. Instead of the nanotriangles, nanoribbon structures were used as shown in the insert. Insert shows the supercell of the nanoribbon with 4 oxygen atoms chemisorbed. The per-atom cohesive energy of oxygen atoms is defined as

$$
E_{c o h}=\left[E_{t}-E_{0}-n \frac{E\left(O_{2}\right)}{2}\right] / n
$$

where $\mathrm{n}$ denotes the number of oxygen atom absorbed to the edge, $E_{\mathrm{t}}, E_{0}$ and $E\left(\mathrm{O}_{2}\right)$ are the total energy of $\mathrm{WS}_{2}$ nanoribbon with oxygen atoms, bare $\mathrm{WS}_{2}$ nanoribbon, and oxygen molecule, respectively. The structure with a higher negative cohesive energy gives rise to a higher stability. As we can see, when number of oxygen atoms is equal to the number of edge tungsten atoms, the cohesive energy reaches its minimum, resulting in the most stable structure. When number of oxygen atoms is over the number of edge tungsten atoms, the structure turns to be less stable. Therefore, the structure with $50 \%$ oxygen coverage (one oxygen atom is chemisorbed by two tungsten atoms, and for every $\mathrm{W}$ atom, two oxygen atoms are connected) at the edge is evaluated to have the highest structural stability. Therefore, in the simulations of the triangle structures, we took this kind of edge configuration. 